\title{
Further Questions for Glashow
}

\author{
Goran Senjanović, reply by Sheldon Lee Glashow
}

In response to "The Yang-Mills Model" (Vol. 5, No. 2).

To the editors:

It is an honor and a pleasure to comment on Sheldon Lee Glashow's magisterial essay on the Yang-Mills model. His essay is all the more special for describing one of the greatest chapters in the history of physics, the story of a beautiful mathematical concept transformed into a theory for all seasons, the Standard Model of particle physics. It was of course Glashow who played a central role in the electroweak part of the story by coming up with his masterpiece, the $\mathrm{SU}(2) \times \mathrm{U}(1)$ gauge theory. And with the added masterstroke from Steven Weinberg, his high-school buddy, as he reminds us, we now have not just a theory of all relevant particle interactions at today's energies, but also a theory of the origin of the masses of elementary particles. Simply, mass turned into a dynamical variable, the knowledge of which enables us to unambiguously predict the associated Higgs boson decay into the relevant particles. And we know with certainty that the $\mathrm{W}$ and $\mathrm{Z}$ bosons, predicted by Glashow, and the third-generation fermions receive their masses from the Higgs mechanism.

To this day, more than 45 years after learning it, I am still in awe of the simplicity and predictivity of this beautiful theory. I came upon the Standard Model at the very beginning of my $\mathrm{PhD}$, when I felt desperate about the dead-end road of that era's particle physics, the dark days of the bootstrap and S-matrix theory, which had complete disregard for the fundamental.

When I look back, I feel that the work of Glashow, Weinberg, and Abdus Salam, together with the quantum chromodynamics part of the tapestry, saved my life in physics. For this reason, I am indebted to the fathers of the Standard Model and to Glashow in particular, and reading this moving narrative of its history brought back a flood of memories.

In short, I love this essay, but I also have some questions and comments.

I was extremely surprised to find out that André Petermann is one of the fathers of the quark picture, a cornerstone of our field, and even more surprised that Glashow mentions this only in passing. It is already mind-boggling that George Zweig and James Bjorken never got the Nobel Prize for their seminal works; after all, Makoto Kobayashi and Toshihide Maskawa got it for predicting more of the same. Now it seems that Petermann should also be included. It would be nice to know more about that.

Glashow remarks that Yang-Mills symmetry is naturally there in the string picture. It is hard to understand why that matters. Should we appreciate Yang-Mills theory more because of that? Or should we appreciate the strings more? Presumably the latter, since the Yang-Mills theory is at the core of high-energy physics, while strings are a wild speculation, to this day phenomenologically unjustified. I should add that Glashow's criticism of string theory over the years has meant a lot to me, making it even more surprising that he should bring up this point.

It is intriguing that Glashow includes the discovery of omega-minus in the list of great achievements of the annus mirabilis, 1964. While one can appreciate the historic role it played, it does not seem to be in the same league as the truly great discoveries on the list. It would be nice to hear more of his arguments for its inclusion.

Glashow brings up the amusing fact that he and Bjorken failed to bring in the Glashow-Iliopoulos-Maiani mechanism in their original paper on the charm. I find it even more amazing that they do not even cite Glashow's historic 1961 paper, the basis of it all. ${ }^{1}$ This has puzzled me for years.

I, for one, never understood the great fascination that Wolfgang Pauli and Arthur Eddington had for the value $\alpha^{-1}=137$. To me, it was a question of numerics and nothing more. We know today that this is just a historical accident since couplings run with energy. It is interesting that Glashow offers no opinion or sentiment on the issue; however, once brought up, it is maybe worth commenting on the energy dependence of couplings.

At the end of the essay, Glashow lists what he believes are some of the central issues left unanswered by the Standard Model. He places the fundamental issue of neutrino mass on an equal footing with rather vague questions, such as why there are three families of fermions, and why the parameters are what they are. Whatever the number of 
families, we could argue that it is a puzzle, but why? After all, we know that three families of quarks lead to a beautiful physics of CP violation. And with regards to the random pattern of masses and mixings-what's wrong with that?

True, for decades many have been arguing that parameters ought to emerge from a simple and predictive principle, but nothing has come of that. I believe that is precisely because it is not a genuine physics question. After all, physics is about correlating apparently different physical processes into a unifying picture, rather than worrying about the values of the parameters of our fundamental theories.

Of course, I could be dead wrong about this. In any case, the main beauty and strength of the Standard Model lies in its predictivity, which stems from it being the minimal electroweak gauge theory. And it is precisely the minimality that led to the only failure of the Standard Model, the prediction of the massless neutrino. Neutrino mass is thus arguably the best door to new physics beyond the Standard Model, which brings me to the next issue.

The essence of the success of the Standard Model, besides its gauge character, is the maximality of parity violation. It is worth recalling that Tsung-Dao Lee and Chen-Ning Yang, in their landmark paper on parity violation, argue that at its most fundamental level parity could be restored. ${ }^{2}$ In any case, it was parity violation that allowed Weinberg to complete Glashow's oeuvre. In order to better appreciate it, try to construct the Higgs version of the Standard Model in an imaginary world of unbroken parity with both left-handed and right-handed quark and lepton doublets. All kinds of problems arise, starting with gauge invariant masses, which are equal for the upper and lower components of the doublets. The only way to split them is through the real Higgs triplet representation, which would leave the $\mathrm{Z}$ boson massless, needing more Higgs. Basically, all predictions would be gone.

In such a world, the neutrino would be massive, a blessing from today's point of view. It is then suggestive that the path to understanding the origin of neutrino mass could lie in understanding the origin of parity breaking, incorporated in the left-right symmetric model. When it comes to neutrino mass, the left-right symmetric model turns out to be self-contained, in analogy with the Standard Model as a theory of charged fermion masses. If neutrinoless double-beta decay is seen and electrons come out righthanded, this would imply new physics such as this one, and it would have to lie tantalizingly close to the reach of the Large Hadron Collider.

These questions aside, Glashow has written an outstanding essay on a historic subject that has shaped particle physics, and in which he has played a key role. I wish there were more such articles nowadays, which touch the core of it all and illuminate it.

\section{Goran Senjanović}

\section{Sheldon Lee Glashow replies:}

Goran Senjanović offers embarrassingly effusive praise for my essay. He asks many sensible questions, some of which I will endeavor to answer. I met André Petermann in 1959 while I was a postdoctoral fellow at the Niels Bohr Institute in Copenhagen and at CERN. Two years earlier, he made the first correct estimate of the two-loop radiative correction to the electron's electric dipole moment, but just three months later, my Harvard classmate Charles Sommerfield succeeded in finding its exact value. Petermann casually mentioned his notion of fractionally charged constituents of nucleons in a brief but otherwise unremarkable article that he submitted to Nuclear Physics in 1963 but which was not published until 1965. In the intervening year, Murray Gell-Mann invented his quarks and George Zweig his equivalent aces. Gell-Mann's paper has since amassed 3,210 citations and Petermann's a mere 15, while Zweig's contribution languishes as unpublished CERN reports. String theories require each and every force of nature to arise from a gauge theory. So they do: strong, weak, electromagnetic, and gravitational! That's very good news, but what about the nasty scalar fields needed for spontaneous symmetry breaking, as well as those called upon for various cosmological purposes?

Why did I credit the 1964 observation of omega-minus baryons by Nick Samios and his team as a great discovery? Gell-Mann and Yuval Ne'eman's flavor SU(3) symmetry scheme was not widely accepted until the omega-minus was found, displaying precisely the properties Gell-Mann had foreseen. It was the tenth member of the spin-3/2 baryon decimet, whose very existence would be demanded by the emerging quark model. Truly, the discovery of the $\mathrm{J} /$ Psi meson was far more significant than that of the omega-minus, but it took place ten years later.

Why did BJ Bjorken and I, while on 1964 sabbaticals in Copenhagen, fail to recognize that our proposal of a fourth charmed quark could enable my electroweak model to apply to all known particles? Why did we not cite my electroweak paper written in Copenhagen four years earlier? And why is it that nobody else devised the Glashow-Iliopoulos-Maiani mechanism until John Iliopoulos and Luciano Maiani joined me at Harvard five years later? These questions puzzle me as well.

Lastly, let me turn to the long-forgotten mystical interpretations of the fine structure constant, alpha. These were not limited to Wolfgang Pauli and Arthur Eddington, but shared with Carl Jung and Arnold Sommerfeld. Max Born, too, had written, "The fact that alpha has just its value of $1 / 137$ is certainly no chance, but is itself a law of nature." 3 Precision data, as well as renormalization group considerations, show that many fundamental constants, including alpha, are energy dependent. At zero energy its value is known to better than parts per billion. Its recip- 
rocal is roughly 137.036 and certainly not an integer. At the electroweak scale, its value rises to about $1 / 127$. It is fitting to end my response to Senjanović by recalling that Petermann and Ernst Stueckelberg are the acknowledged fathers of the renormalization group. ${ }^{4}$

Goran Senjanovic is a theoretical physicist at the Abdus Salam International Centre for Theoretical Physics.

Sheldon Lee Glashow is a Nobel Laureate, Higgins Professor of Physics, emeritus, at Harvard University, and University Professor, emeritus, at Boston University.
1. Sheldon Lee Glashow, "Partial-Symmetries of Weak Interactions," Nuclear Physics 22, no. 4 (1961): 579-88, doi:10.1016/0029-5582(61)90469-2.

2. Tsung-Dao Lee and Chen-Ning Yang, "Question of Parity Conservation in Weak Interactions," Physical Review 104, no. 1 (1956): 254-58, doi:10.1103/PhysRev.104.254.

3. Max Born, "Tainstvennoe chislo 137 (The Mysterious Number 137)," Uspekhi Fizicheskikh Nauk 16, no. 6 (1936): 697-729.

4. André Petermann and Ernst Stueckelberg, "La Normalisation des constantes dans la théorie des quanta (Normalization of Constants in Quantum Theory)," Helvetica Physica Acta 26 (1953): 499-550. 